\title{
Computation in Linear Algebraic Monoids
}

\section{Zhenheng Li*}

Department of Mathematical Sciences, University of South Carolina Aiken Aiken, SC 29801, USA

This paper is intended to very briefly introduce some computation problems in linear algebraic monoids. The theory of linear algebraic monoids was initiated independently in 1980 by Mohan Putcha (North Carolina State University) and Lex Renner (The University of Wester Ontario). This theory is a natural blend of algebraic groups, torus embeddings, and semigroups. It is a very active and fruitful research area in mathematics. Many other mathematicians work on this field or related areas: M. Brion, M. Can, Y. Cao, E. Godelle, W. Huang, Zhuo Li, J. Okninski, A. Rittatore, L. Solomon, E. B. Vinberg [1-3] etc. The fundamental developments since 1980 and a systematic description of the theory can be found in [3-5].

There are a lot of computation problems in the theory of linear algebraic monoids, such as the orders of the Renner monoids, generating functions of the Renner monoids, cardinalities of monoids of Lie type, conjugacy classes in Renner monoids and H-polynomials.

A general formula for calculating the orders of Renner monoids of classical types A, B, C, D was obtained in [6]. The structure of a Renner monoid $\mathrm{R}$ is completely determined by its unit group $\mathrm{W}$ and a cross section lattice $\Lambda$ of $\mathrm{R}$; it turns out that $\mathrm{R}=\mathrm{W} \Lambda \mathrm{W}$. Using this structure in the same paper, the orders of exceptional Renner monoids were computed using Mathematica, as well. The Renner monoid plays the same role in reductive monoids as the Weyl group does in reductive groups. We refer the reader to [7] for Renner monoids in general and to $[8,9]$ for classical Renner monoids.

Generating functions of classical Renner monoids were studied in $[10,11]$. Borwein, Rankin, and Renner first obtained the generating functions for the classical Renner monoids of type A and found their connections to Laguerre polynomials and Stirling numbers of the second kind. Y. Cao, Zhuo Li [11] and the author investigated the generating functions of the Renner monoids of types B; C and D; similar connections to Laguerre polynomials were found. The generating functions of the dual symplectic Renner monoids were obtained in [12].

Yan [13] obtained a formula for the orders of monoids of Lie type based on how the monoids are constructed. He then used computers to calculate the order of the monoid of $\mathrm{n} \times \mathrm{n}$ matricides over a finite field. However, his formula cannot compute the orders of finite reductive monoids $\mathrm{M}$ in general, even though every finite reductive monoid is a finite monoid of Lie type. Four different formulas for computing the orders of $\mathrm{M}$ in terms of its type map were given in [14]. These formulas allow computer programs such as GAP, Maple and Mathematica to play important roles in finding the number of elements in $M$ which is large and involves complex computation that cannot be handled by hands.

Renner [15] established an enumerative theory of finite reductive monoids using a length function on Renner monoids. Recently, in [1,1618] Can and Renner gave a systematic description of H-polynomials of reductive monoids, whose whole point is to investigate the orders of finite reductive monoids.

The conjugacy classes of symplectic Renner monoids $\mathrm{R}$ were investigated in [2] by introducing a new concept, the symplectic partition of a positive integer. Two elements are conjugate if and only if they have the same symplectic cycle-link type. In addition, an algorithm for finding the number of conjugacy classes was given, and a formula for calculating the order of each class was shown in the above paper. We refer the reader to [19-21] and the references there for a systematic study of conjugacy classes of reductive monoids; the conjugacy on the Weyl group and on finite groups of Lie type can be seen in [22,23].

The conjugacy classes of any Renner monoid were investigated in [24]. One of its main results is that the conjugacy of two elements in the monoid is described by using the action of the centralizer of idempotents on certain parabolic subgroups of the Weyl group. Using this result and with the help of GAP, it is shown that there are 1882 conjugacy classes in the first basic Renner monoids of type E6 [25]. The conjugacy classes of other basic Renner monoids of type E6 can be found the same way. Zhuo $\mathrm{Li}$ and the author have computed all the conjugacy classes of the Renner monoids of other types $\left(A_{n}, B_{n}, C_{n}\right.$, $\mathrm{D}_{\mathrm{n}}, \mathrm{F}_{4}, \mathrm{G}_{2}$ ) for $\mathrm{n} \leq 5$. For those who are interested in conjugacy on semi groups in general, see [26-30].

\section{References}

1. Can M, Renner L (2008) H-polynomials and rook polynomials. Int J Algebra Comput 18: 935-949.

2. Cao Y, Li Z (2010) Conjugacy classes in the symplectic Renner monoid Journal of Algebra 324: 1940-1951.

3. Solomon $L$ (1995) An introduction to reductive monoids, Semigroups, Forma Languages and Groups. J Fountain, ED., Kluwer Academic Publishers 295-352.

4. Putcha M (1988) Linear Algebraic Monoids, London Math. Soc. Lecture Note Series 133, Cambridge University Press.

5. Renner L (2005) Linear Algebraic Monoids, Series: Encyclopedia of Mathematical Sciences. Springer-Verlag 134.

6. Li Z, Cao Y (2006) Orders of the Renner monoids. Journal of Algebra 301 344-359.

7. Renner $L$ (1986) Analogue of the Bruhat decomposition for algebraic monoids $\mathrm{J}$ of Algebra 101: 303-338.

8. Li Z (2001) The Renner monoids and cell decompositions of classical algebraic monoids. The University of Western Ontario, Canada.

9. Li Z, Renner L (2003) The Renner monoids and cell decompositions of the symplectic algebraic monoids. International Journal of Algebra and Computation 13: 111-132.

10. Borwein D, Rankin S, Renner L (1989) Enumeration of Injective Partia Transformations. Discrete Mathematics 73: 291-296.

11. Li Z, Zhuo Li, Cao Y (2006) Enumeration of symplectic and orthogonal injective partial transformations. Discrete Mathematics 306: 1781-1787.

*Corresponding author: Zhenheng Li, Department of Mathematical Sciences, University of South Carolina Aiken Aiken, SC 29801, USA, Tel: 1 803-648-6851; E-mail: zhenhengl@usca.edu

Received January 01, 2014; Accepted January 02, 2014; Published January 05 2014

Citation: Li Z (2014) Computation in Linear Algebraic Monoids. J Appl Computat Math 3: e135. doi:10.4172/2168-9679.1000e135

Copyright: $\odot 2014 \mathrm{Li}$ Z. This is an open-access article distributed under the terms of the Creative Commons Attribution License, which permits unrestricted use distribution, and reproduction in any medium, provided the original author and source are credited. 
12. Li Z, Torkian B (2009) Generating functions of the dual symplectic Rook monoid. Global Journal of Pure and Applied Mathematics 5: 95-100

13. Yan R (1995) On orders of monoids of Lie type, Semigroups. Papers from the International Conference on Semigroups and Its Related Topics, Kunming, China, Springer, Singapore.

14. Li Y, Cao (2008) Orders of finite reductive monoids, arXiv:0812.3105 [math GR].

15. Renner L (1995) Finite reductive monoids, Semigroups, Formal Languages and Groups. J Fountain, Kluwer Academic Publishers, USA.

16. Renner L (2011) Weyl groups, descent systems and Betti numbers. Rocky Mountain Journal of Mathematics 41: 1329-1359.

17. Renner L (2009) Rationally smooth algebraic monoids. Semi group Forum 78 : 384-395.

18. Renner L (2008) The H-polynomial of a semi simple monoid. J of Algebra 319: 360-376.

19. Putcha M (1987) Conjugacy classes in algebraic monoids. Trans Amer Math Soc 303: $529-540$.

20. Putcha M (1994) Conjugacy classes in algebraic monoids II. Canad J Math 46: 648-661.
21. Renner L (1988) Conjugacy classes of semisimple elements, and irreducible representations of algebaic monoids. Comm Alg 16: 1933-1943.

22. Carter R (1972) Conjugacy classes in the Weyl group. Compositio Mathematica 25: $1-59$.

23. Carter R (1985) Finite groups of Lie type: Conjugacy classes and complex characters Wiley.

24. Li Z, Cao Y (2013) Conjugacy classes of Renner monoids. Journal of Algebra 374: 167-180.

25. Li Z, Zhuo Li (2013) The 1882 conjugacy classes of the first basic Renner monoid of type E6. Journal of Applied and Computing Mathematics.

26. Ganyushkin O, Mazorchuk V (2009) Classical finite transformation semi groups. Springer.

27. Kudryavtseva G, Mazorchuk V (2009) On three approaches to conjugacy in semi groups. Semigroup Forum 1: 14-20.

28. Kudryavtseva G, Mazorchuk V (2007) On conjugation in some transformation and Brauer-type semi groups. Publ Math Debrecen 12: 19-43.

29. Lipscomb S (1996) Symmetric inverse semigroups, American Mathematica Society, Providence, RI.

30. Munn W (1957) The characters of the symmetric inverse semigroup. Proc Camb Phil Soc 53: 13-18. 